\title{
FRAIL scale as a predictor of complications and mortality in older patients undergoing reconstructive surgery for non-melanoma skin cancer
}

\author{
LUIGI VALDATTA $^{1 *}$, GIANPAOLO PERLETTI ${ }^{1,2^{*}}$, FRANCESCA MAGGIULLI $^{3 *}$, \\ FEDERICO TAMBORINI $^{3}$, IGOR PELLEGATTA ${ }^{1}$ and MARIO CHERUBINO ${ }^{1}$ \\ ${ }^{1}$ Department of Biotechnology and Life Sciences, Section of Medical and Surgical Sciences,
} University of Insubria, I-21100 Varese, Italy; ${ }^{2}$ Department of Human Structure and Repair, Faculty of Medicine and Medical Sciences, Ghent University, B-9000 Ghent, Belgium; ${ }^{3}$ Plastic and Reconstructive Surgery Complex Unit, ASST Sette Laghi Varese, I-21100 Varese, Italy

Received March 20, 2018; Accepted August 22, 2018

DOI: $10.3892 / \mathrm{ol} .2018 .9568$

\begin{abstract}
The aim of the present study was to determine the association between preoperative frailty and the onset of surgical complications in patients diagnosed with massive non-melanoma skin cancer subjected to plastic and reconstructive surgery. A retrospective analysis was performed on a cohort of 587 patients with non-melanoma skin cancer, selected on the basis of specific inclusion criteria, who were subjected to plastic and reconstructive surgery between 2005 and 2014. Frailty was scored using the FRAIL index, whereas postoperative complications were classified according to Clavien-Dindo criteria. By binary logistic regression, the odds and probabilities of complications were calculated as a function of increasing values of the FRAIL index. Two different logistic models were created, comparing absent/mild (Clavien grades 1st and 2nd) vs. moderate/severe complications or mortality (Clavien grades 3rd-5th; model A), or absent/mild/moderate complications (Clavien grades 1st-3rd) vs. severe complications or mortality (Clavien grades 4th and 5th; model B). The FRAIL index was an accurate predictor of surgical complications or mortality, with significant odds ratios and goodness of fit. In model A, FRAIL scores 4 and 5 were the most critical predictors of moderate/severe complications or mortality (37 and
\end{abstract}

Correspondence to: Professor Gianpaolo Perletti, Department of Biotechnology and Life Sciences, Section of Medical and Surgical Sciences, University of Insubria, Via Manara 7, 21052 Busto A, I-21100 Varese, Italy

E-mail: gianpaolo.perletti@uninsubria.it

${ }^{*}$ Contributed equally

Key words: frailty, non-melanoma skin cancer, postoperative complications, logistic regression, postoperative mortality, plastic surgery, reconstructive surgery
94\% probability, 0.6 and 17.3 odds, respectively), compared to score 3 (2\% probability, 0.02 odds) or lower. In model B, FRAIL score 5 was the most critical predictor of severe complications or mortality, as it was associated with a $74.6 \%$ probability and 2.93 odds for these events. In conclusion, increasing FRAIL scores were associated with worsening surgical outcomes for patients with non-melanoma skin cancer undergoing plastic/reconstructive surgery. A low rate of surgical complications was observed in pre-frail and frail patients up to FRAIL score 3.

\section{Introduction}

The European population is progressively aging, due to increased life expectancies and decreased birth rates, caused by the phenomenon known as 'demographic transition'. The lengthening of life has re-shaped the understanding and classification of the elderly population. Hence, 'elders' (65 years or older) are distinguished from 'great elders' (80 years or older). At 80 years of age, average life expectancy for women is about 10 and 8 years for men. According to the European Commission, it is predicted that by year 2020 a quarter of the European population will be 60 years or older (https://ec.europa.eu/research/social-sciences/pdf/policy_ reviews/kina26426enc.pdf).

As with many types of cancer, the prevalence of non-melanoma skin cancer increases with age, and about $50 \%$ of cases are diagnosed in individuals older than 65 years (1). A 2012 survey showed that, in continental Europe, Switzerland and Italy showed the highest incidence rates at approximately 70-100,000 person-year (2). A diagnosis of cancer in older individuals should be managed with curative intent despite advanced age, though targeted interventions should be initiated after considering age-related comorbidities and any other element-including frailty-which may contraindicate conventional surgery or even adjuvant treatments.

Patient frailty has been a field of increasing study in the last two decades. Such interest has been fostered by the increased rate of surgical indications for older individuals. Although 
much research has been devoted to this subject, no universal agreement has been reached concerning the criteria used for describing frailty. Nevertheless, there is agreement regarding the general definition of frailty as an age-dependent biological status characterized by reduced stress resistance, secondary to the cumulative decline of various physiological systems and related to comorbidity, disability, risk of institutionalization and mortality.

Several validated diagnostic tools are available to classify and to measure increasing frailty (3-5).

The concept of frailty in elderly individuals has been associated with surgery-related adverse events. Several studies have recently correlated preoperative frailty with poor surgical results or with the occurrence of complications and adverse events during or after surgery (6-8). Systematic review of the literature performed in year 2016 indicates that frailty in surgical patients can predict post-operative mortality, complications, and prolonged hospitalization (9). Notably, this association was shown to be consistent across different frailty instruments and regardless of the type of surgery performed. Such association has also been thoroughly investigated in the frame of a study involving a population of about a million patients who underwent surgical operations of various specialties. It was shown that a modified, 11-item version of the Canadian Study of Health and Aging Frailty Index (CSHA-FI) correlated significantly with both mortality and morbidity across all surgical specialties (10).

Despite such impressive collection of recent data focusing on many surgical specialties, evidence about the association between frailty and surgical complications in the field of plastic and reconstructive surgery is still very limited. To investigate this association in non-melanoma skin cancer patients with indication for various plastic and reconstructive surgical procedures, we designed a retrospective analysis on a cohort of 587 elderly patients consecutively referring to a single Northern-Italian university hospital.

\section{Patients and methods}

Patients-demographics, surgical indications and inclusion criteria. We conducted a retrospective study involving patients of age 65 years or older who referred to the University of Insubria/Macchi Foundation Hospital in Varese, Italy, for elective plastic surgery for excision of massive non-melanoma skin cancers between January 2005 and December 2014 (11).

Participants underwent a standardized preoperative interview and frailty assessment by a surgeon. Demographic information, a comprehensive medical history and the current therapies were obtained during the interview. All patients provided written informed consent about the use of their anonymous clinical data for research purposes.

Inclusion criteria were an age $>65$ years, any frailty status, and surgical indications for non-melanoma skin cancer excision, associated with skin grafting or local/distant flap surgery.

Exclusion criteria for the study were a history of limb amputation or other severe complications of diabetes, ongoing or previous (4 months) therapy with drugs that may act as confounders in the evaluation of frailty (e.g., sedatives, opioid analgesics, antipsychotics, and chemotherapy) and the lack of data concerning frailty or post-surgery complications.
Classification of patients: Frailty and surgical complications. The present study was designed to investigate the association between frailty, assessed with the FRAIL score (4) adopted as predictor (explanatory variable), and the grade of severity of surgical complications, assessed according to the Clavien-Dindo classification (12).

The FRAIL score, validated by Morley and coworkers, is based on a phenotypic model including five items contributing to increasing patient frailty: Fatigue, Resistance, Ambulation, Illnesses, Loss of weight (4). The Frail scale ranges from 0 to 5 (i.e., one point for each added component, $0=$ best to $5=$ worst), and indicates a frail (3-5), pre-frail (1-2), or robust (0) health status.

The classification system validated by Dindo et al is based on a scale including five grades (1st to 5th), indicating increasing severity of surgical complications (13). Briefly, first grade complications require no treatment or minor drug or physical treatment. Second grade complications require a major pharmacological intervention or transfusion. Third grade complications require surgical or endoscopic interventions, whereas fourth grade events are life-threatening and require intensive care management. The death of the patient is a fifth-grade event.

Frailty and surgical complication data were collected up to 6 months after surgery.

Sample size. The minimum sample size for this study, calculated according to Whittemore (14), is $425(1-\beta=0.95)$, $349(1-\beta=0.9)$, and $267(1-\beta=0.8)$ patients, given a minimum acceptable odds-ratio equal to $3.0, \alpha=0.05$, and a probability of the event at the median value of the covariate (FRAIL score=3) equal to 0.02 (two-tailed test). The minimum acceptable odds-ratio was estimated conservatively, based on published data showing an odds-ratio equal to 4.38 (95\% CI: 1.3-14.4), for a logistic regression model describing the odds for surgical complications according to Clavien-Dindo in reconstructive surgery in oncology as function of a frailty index similar to the FRAIL score (15)

Statistical analysis. Data were analyzed by co-author GP, who was blinded to the identity and clinical condition of each patient, and was not involved in data collection or frailty assessment.

Statistical analysis was performed using the Rstudio integrated development environment for ' $R$ '.

In the ' $\mathrm{R}$ ' environment, the stats package was used to perform the Kruskal-Wallis test.

Two binary logistic regression models were generated to estimate the odds and probability of surgical complications or death as function of the FRAIL score predictor. In model A, we dichotomized absent/mild complications (Clavien-Dindo naught, 1 st and 2 nd grades) vs. moderate/severe complications or death (Clavien-Dindo 3rd, 4 th and 5 th grades). In model B, we dichotomized absent $/ \mathrm{mild} /$ moderate surgical complications (Clavien-Dindo naught, 1st, 2nd and 3rd grades) vs. severe complications or death (Clavien-Dindo 4 th and 5 th grades).

The null hypothesis for logistic regression was that moderate/severe complications or death (in model A), or severe complications or death (in model B) were not significantly associated with the predictor. 
Table I. Demographics, clinical characteristics and surgical indication of patients, based on their baseline FRAIL score.

\begin{tabular}{|c|c|c|c|c|c|c|c|}
\hline FRAIL score, baseline & 0 & 1 & 2 & 3 & 4 & 5 & Total \\
\hline Total patients & 43 & 71 & 99 & 207 & 137 & 30 & 587 \\
\hline Mean age & 80.7 & 80.6 & 81.6 & 80.7 & 78.9 & 78.6 & 80.3 \\
\hline \multicolumn{8}{|l|}{ Sex } \\
\hline Men, $(\%)$ & $33(76.7 \%)$ & $46(64.7 \%)$ & $63(63.4 \%)$ & $107(51.7 \%)$ & $83(60.5 \%)$ & $11(36.6 \%)$ & $343(58.4 \%)$ \\
\hline Women, $(\%)$ & $10(33.3 \%)$ & $25(35.3 \%)$ & $36(36.3 \%)$ & $100(48.3 \%)$ & $54(39.5 \%)$ & $19(63.4 \%)$ & $244(41.6 \%)$ \\
\hline \multicolumn{8}{|l|}{ Diagnosis } \\
\hline Epitheliomas & 43 & 65 & 99 & 202 & 137 & 30 & 576 \\
\hline $\begin{array}{l}\text { Non-epitheliomas (cylindroma, } \\
\text { sarcoma, Merkel's cell cancer, } \\
\text { fybroxanthoma) }\end{array}$ & 0 & 6 & 0 & 5 & 0 & 0 & 11 \\
\hline \multicolumn{8}{|l|}{ Indication } \\
\hline Simple reconstruction & 15 & 25 & 26 & 56 & 49 & 11 & 182 \\
\hline $\begin{array}{l}\text { Medium complexity } \\
\text { reconstruction (skin graft, } \\
\text { local flap, artificial skin graft) }\end{array}$ & 28 & 45 & 71 & 148 & 87 & 19 & 398 \\
\hline $\begin{array}{l}\text { Complex reconstruction } \\
\text { (microsurgery) }\end{array}$ & 0 & 1 & 2 & 3 & 1 & 0 & 7 \\
\hline
\end{tabular}

Simple binary logistic regression analysis was performed using the car, rcompanion, and lmtest packages. The Hosmer and Lemeshow goodness-of-fit test was performed with the Resource Selection package, whereas the Nagelkerke pseudo $(\psi)-\mathrm{R}^{2}$ was calculated using the rcompanion package.

For statistical analysis only two-tailed tests were performed, 95\% confidence intervals were calculated, and the conditional probability of a type I error, in the presence of a true null hypothesis, was set at $<0.05$.

\section{Results}

From our clinical database, we identified a total of 587 subjects who met our inclusion criteria. Patients' age ranged from 65 to 100 years with a median age of 81 years and an interquartile range (IQR) of 12 (mean age: 80.3 years). The median FRAIL score of the population was 3 , with an IQR of 2. Table I summarizes demographic and clinical baseline data of patients.

To evaluate whether patients with different frailty status showed differences in the severity of surgical complications, we stratified our patient population in 6 groups based on the individual score of the FRAIL scale (0 to 5). The Kruskal-Wallis test was performed considering the FRAIL score as independent variable and the surgical complication severity score (Clavien-Dindo 1st to 5th grade) as dependent variable. Analysis rejected the null hypothesis that the median Clavien-Dindo complication scores of the 6 groups are equal, as rank differences in these groups were statistically significant $\left(\chi^{2}=286.4, P<0.00001\right)$.

Table II summarizes the logistic function coefficients of models A and B, and the odds ratios. Due to the different placement of Clavien-Dindo grade 3 complications, the odds ratio (OR) for complications or death at each unit increase of the FRAIL score is higher in model B $(\mathrm{OR}=40.9)$ than in model A (OR=29.2).
Table III shows the significance assessments of both models and the goodness-of-fit parameters. The Wald test and the likelihood ratio test were highly significant $(\mathrm{P}<0.00001$ for both tests and for either model), and the pseudo- $\mathrm{R}^{2}$ value and Hosmer and Lemeshow test confirmed the goodness-of-fit of both models. In particular, the Nagelkerke pseudo- $\mathrm{R}^{2}$ in both models A and B suggests a strong correlation between frailty and surgical complications, and the very high 'P' value of the Hosmer and Lemeshow test confirms the null hypothesis that the number of expected complications-assessed when the patient cohort was partitioned into 10 groups of approximately similar size-is not significantly different from the number of complications observed in the whole logistic model.

Table IV lists the odds and probabilities of surgical complications calculated at each level of the FRAIL score predictor. In both models evaluated, logistic regression analysis shows that increasing FRAIL index scores were associated with increasing probabilities and odds for moderate/severe complications or death (model A), or severe complications or death (model B). Up to a FRAIL score of 3, the probability of complications or death is $1.9 \%$ in model $\mathrm{A}$ and $1.7 \%$ in model B. For the frailest patients (FRAIL score $=5$ ) in model A the probability for moderate/severe complications or death is $94.5 \%$ and the odds is 17.3 , whereas in model B the probability for severe complications or death is $74.6 \%$ and the odds is 2.9 .

\section{Discussion}

Research focusing on the association between frailty and postoperative morbidity and mortality in various surgical subspecialties has gained great momentum in the last few years (16-24). The results of our study add to the existing evidence that frailty can be an accurate predictor of the probability and odds of surgical complications or death in patients undergoing plastic and reconstructive surgery. These findings 
Table II. Logistic regression models A and B for complications or death as function of preoperative FRAIL score.

\begin{tabular}{lcr}
\hline Logistic model parameters & $\begin{array}{c}\text { Model A 3rd/4th/5th grade } \\
\text { vs. naught/1st/2nd grade }\end{array}$ & $\begin{array}{c}\text { Model B 4th/5th grade } \\
\text { vs. naught/1st/2nd/3rd grade }\end{array}$ \\
\hline Intercept \pm standard error & $-14.02 \pm 1.64, \mathrm{P}<0.00001$ & $-17.48 \pm 2.15, \mathrm{P}<0.00001$ \\
Coefficient \pm standard error & $3.37 \pm 0.42, \mathrm{P}<0.00001$ & $3.71 \pm 0.49, \mathrm{P}<0.00001$ \\
Odds ratio & $29.23(95 \% \mathrm{CI}: 14.15$ to 74.48$)$ & $40.92(95 \% \mathrm{CI}: 16.84$ to 116.07$)$ \\
$\mathrm{EL}_{50}{ }^{\mathrm{a}}$ & 4.15 & 4.71 \\
\hline
\end{tabular}

${ }^{\mathrm{a}}$ Grade of Clavien-Dindo surgical complications. ${ }^{\mathrm{b}} \mathrm{EL}_{50}$, median effective level, i.e., FRAIL score associated with $50 \%$ probability of (i) moderate/severe complications or death (model A) or (ii) severe complications or death (model B). CI, confidence interval.

Table III. Predictor significance tests and goodness-of-fit parameters of logistic regression models A and B.

\begin{tabular}{lcc}
\hline Test & $\begin{array}{c}\text { Model A 3rd/4th/5th grade } \\
\text { vs. naught/1st/2nd grade }\end{array}$ & $\begin{array}{c}\text { Model B 4th/5th grade } \\
\text { vs. naught } / 1 \text { st } / 2 \text { nd } / 3 \text { rd grade }\end{array}$ \\
\hline Wald & $\chi^{2}=65.68, \mathrm{P}<0.00001$ & $\chi^{2}=57.27, \mathrm{P}<0.00001$ \\
Likelihood ratio test & $\chi^{2}=244.49, \mathrm{P}<0.00001$ & $\chi^{2}=141.03, \mathrm{P}<0.00001$ \\
Hosmer \& Lemeshow & $\chi^{2}=0.17, \mathrm{P}=0.99$ & $\chi^{2}=0.47, \mathrm{P}=0.99$ \\
Nagelkerke & $\psi \mathrm{R}^{2}=0.61$ & $\psi \mathrm{R}^{2}=0.62$ \\
\hline
\end{tabular}

${ }^{\mathrm{a}}$ Grade of Clavien-Dindo surgical complications.

are substantiated by the results of logistic regression analysis and by the assessment of the goodness-of-fit of the logistic models therefrom generated.

Our results are congruent with a very recent study by Abt and coworkers, performed retrospectively on 266 patients who underwent head/neck regional or free flap reconstructions (15). The Abt study focused mainly on the occurrence of Clavien-Dindo grade IV complications based on a 15-item modified frailty index. In the Abt study, about $8 \%$ of patients had Clavien-Dindo 4th grade complications or death, compared to $6 \%$ in the present study. In the same study, multivariate logistic regression analysis showed that frailty was associated with Clavien-Dindo 4th grade complications for all flap procedures, with a significant odds ratio of 4.38 (15). Notably, Abt and coworkers demonstrated that frailty is the best predictor of surgical complications in flap reconstruction compared to other indicators, like for example comorbidity rates. A main difference between our plastic surgery study and the Abt study is that the latter focused on the odds/probability of onset of Clavien-Dindo 4th grade complications alone, whereas in our research 4th grade complications were associated in model A with grades 3 rd and 5 th, and in model $\mathrm{B}$ with grade 5 th complications alone. The choice of this dichotomization strategy for our study was based on the fact that 3rd grade complications comprise conditions showing diverse severity; thus, we modeled two different scenarios, as we deemed interesting to explore the association of 3rd grade complications with milder or more severe ones. This appears to be common practice, since in several studies based on logistic regression, Clavien-Dindo grade 3 complications are grouped together with higher grade complications (e.g., 6,8).
It is virtually impossible to compare the odds ratios resulting from this study with those described in other published studies correlating frailty and surgical complications by logistic regression. This is mainly due to unique study characteristics like the nature of the diseases, the wide variety of surgical specialties and techniques, the degree of invasiveness of the various procedures, the selection of patients, the frail scale adopted, the type of logistic regression model, the adjustments implemented therein, and the inclusion/exclusion of death as a study endpoint. Nevertheless, our study is in full agreement with the general published evidence that a pre-surgery frailty status correlates positively with the odds for postoperative complications.

In conclusion, in this study we observed a low rate of surgical complications in pre-frail and frail patients up to FRAIL score 3. Moreover, the FRAIL index validated by Morley and coworkers (4) was an accurate predictor of surgical complications or death, with significant odds ratios and goodness of fit. In model A, FRAIL scores 4 and 5 are the most critical predictors of moderate/severe complications or death (37 and 94\% probability, respectively), compared to score 3 ( $2 \%$ probability) or lower. In model B, FRAIL score 5 is the most critical predictor of severe complications or death, as it is associated with a $74.6 \%$ probability and 2.93 odds for these events.

A possible limitation to the present study is that in our older patient population deaths for any cause may confound deaths specifically related to surgery or surgical sequelae. A second limitation may be represented by the prevalence in elderly patients of diagnosed or undiagnosed comorbidities that may not directly contribute to the expression of phenotypic frailty 


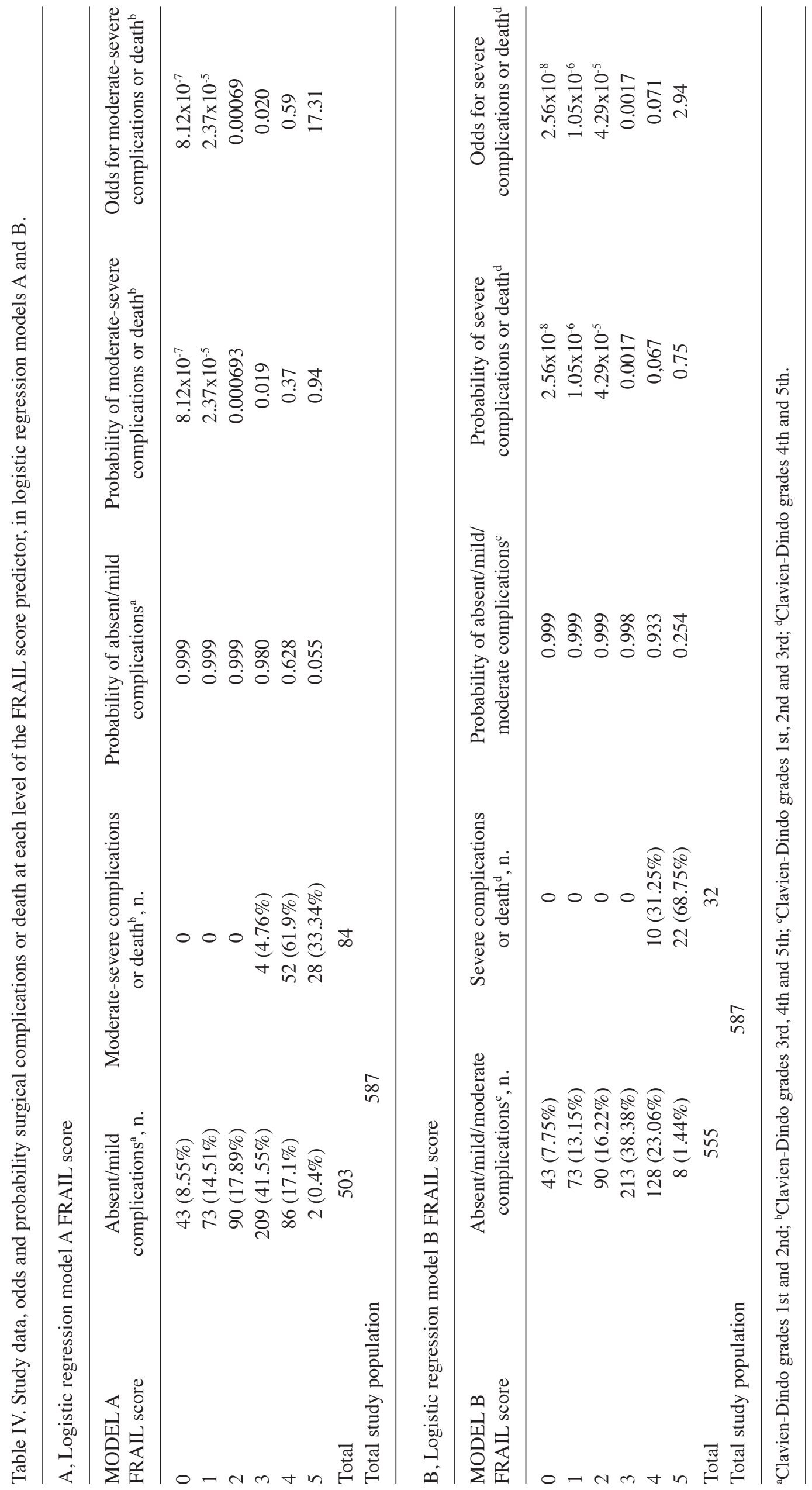


as assessed with the FRAIL scale, but that may nevertheless concur to the onset of surgical complications.

An intrinsic limit to the present clinical study is its retrospective design, which restricted our capability in characterizing some aspects of the frail phenotype of included patients. Although Morley's FRAIL scale (4) was the best available tool for extracting frailty data from our clinical database, the retrospective design of the study did not allow the assessment of important elements contributing to frailty, such as the cognitive fitness and the nutritional status of patients. We plan to include these items in future prospective studies. In fact, the cognition and nutrition items are part of the Edmonton frailty scale, albeit to a limited extent (25). If the Edmonton scale is considered insufficient to perform an in-depth assessment of the cognitive and nutritional status of patients, well-established tools such as the Mini Mental State Examination (MMSE) (26) and the Mini Nutritional Assessment (MNA) (27) questionnaires can be used to complement the Morley FRAIL index or the Edmonton scale.

\section{Acknowledgements}

The authors would like to thank Professor Giovanni Bernardini (Department of Biotechnology and Life Sciences, University of Insubria, Varese, Italy) for promoting collaboration between the surgeons and scientists involved in the present study. The authors would also like to thank Ms. Louise Beckers (Ghent University School of Medicine, Ghent, Belgium) for her assistance in data collection and analysis.

\section{Funding}

No funding was received.

\section{Availability of data and materials}

Single patient data are exclusive legal property of patients, and cannot be provided or disclosed. Global data and study statistics are available upon request to the corresponding author, but must be authorized by the principal investigator (Professor Luigi Valdatta).

\section{Authors' contributions}

LV, FM, FT, IP and MC performed surgery and clinical workup on all enrolled patients and revised the final draft of the article. LV conceived this clinical study. FM extracted study data from all clinical records of patients and prepared the database. GP drafted the clinical protocol to be submitted to the hospital Ethics Committee, performed the statistical analysis of the study and drafted the manuscript.

\section{Ethics approval and consent to participate}

All procedures performed in the present study involving human participants were in accordance with the ethical standards of the institutional research committee and with the 1964 Helsinki declaration and its later amendments. Although for this type of study formal consent of single patients is not required according to Italian laws, all patients signed a written informed consent, authorizing anonymous disclosure and publication of all clinical data, including photographs, for research purposes. The protocol of the study was approved on February 13th, 2018 by the Ethics Committee of the ASST Sette Laghi, Varese, Italy. The ethics committee also provided consent to publication of study data.

\section{Patient consent for publication}

All patients provided written informed consent.

\section{Competing interests}

The authors declare that they have no competing interests.

\section{References}

1. Dacosta Byfield S, Chen D, Yim YM and Reyes C: Age distribution of patients with advanced non-melanoma skin cancer in the United States. Arch Dermatol Res 305: 845-850, 2013.

2. Lomas A, Leonardi-Bee J and Bath-Hextall F: A systematic review of worldwide incidence of non-melanoma skin cancer. $\mathrm{Br}$ J Dermatol 166: 1069-1080, 2012.

3. Hogan DB, MacKnight C and Bergman H; Steering Committee, Canadian Initiative on Frailty and Aging: Models, definitions, and criteria of frailty. Aging Clin Exp Res 15 (3 Suppl): S1-S29, 2003.

4. Morley JE, Malmstrom TK and Miller DK: A simple frailty questionnaire (FRAIL) predicts outcomes in middle aged African Americans. J Nutr Health Aging 16: 601-608, 2012.

5. Dasgupta M, Rolfson DB, Stolee P, Borrie MJ and Speechley M: Frailty is associated with postoperative complications in older adults with medical problems. Arch Gerontol Geriatr 48: 78-83, 2009.

6. Chimukangara M, Frelich MJ, Bosler ME, Rein LE, Szabo A and Gould JC: The impact of frailty on outcomes of paraesophageal hernia repair. J Surg Res 202: 259-266, 2016.

7. Makary MA, Segev DL, Pronovost PJ, Syin D, Bandeen-Roche K, Patel P, Takenaga R, Devgan L, Holzmueller CG, Tian J and Fried LP: Frailty as a predictor of surgical outcomes in older patients. J Am Coll Surg 210: 901-908, 2010.

8. Park JS, Bateni SB, Bold RJ, Kirane AR, Canter DJ and Canter RJ: The modified frailty index to predict morbidity and mortality for retroperitoneal sarcoma resections. J Surg Res 217: 191-197, 2017.

9. Lin HS, Watts JN, Peel NM and Hubbard RE: Frailty and post-operative outcomes in older surgical patients: A systematic review. BMC Geriatr 16: 157, 2016.

10. Velanovich V, Antoine H, Swartz A, Peters D and Rubinfeld I: Accumulating deficits model of frailty and postoperative mortality and morbidity: Its application to a national database. J Surg Res 183: 104-110, 2013.

11. Cherubino M, Scamoni S, Maggiulli F, Pellegatta I and Valdatta L: Use of human fibrin sealant in large split thickness skin grafting after massive skin tumour excision. Minerva Chir 68: 119-121, 2013

12. Clavien PA, Barkun J, de Oliveira ML, Vauthey JN, Dindo D, Schulick RD, de Santibañes E, Pekolj J, Slankamenac K, Bassi C, et al: The Clavien-Dindo classification of surgical complications: Five-year experience. Ann Surg 250: 187-196, 2009.

13. Dindo D, Demartines N and Clavien PA: Classification of surgical complications: A new proposal with evaluation in a cohort of 6336 patients and results of a survey. Ann Surg 240: 205-213, 2004.

14. Whittemore AS: Sample size for logistic regression with small response probability. J Am Stat Assoc 76: 27-32, 1981.

15. Abt NB, Xie Y, Puram SV, Richmon JD and Varvares MA: Frailty index: Intensive care unit complications in head and neck oncologic regional and free flap reconstruction. Head Neck 39: $1578-1585,2017$.

16. Karam J, Tsiouris A, Shepard A, Velanovich V and Rubinfeld I: Simplified frailty index to predict adverse outcomes and mortality in vascular surgery patients. Ann Vasc Surg 27: 904-908, 2013. 
17. Kolbe N, Bakey S, Louwers L, Blyden D, Horst M, Falvo A, Patton J and Rubinfeld I: Predictors of Clavien 4 complications and mortality after necrosectomy: Analysis of the NSQIP database. J Gastrointest Surg 19: 1086-1092, 2015.

18. Kolbe N, Carlin AM, Bakey S, Louwers L, Horst HM and Rubinfeld I: Assessing risk of critical care complications and mortality in the elective bariatric surgery population using a modified frailty index. Obes Surg 25: 1401-1407, 2015.

19. Melin AA, Schmid KK, Lynch TG, Pipinos II, Kappes S, Longo GM, Gupta PK and Johanning JM: Preoperative frailty Risk Analysis Index to stratify patients undergoing carotid endarterectomy. J Vasc Surg 61: 683-689, 2015.

20. Levy I, Finkelstein M, Bilal KH and Palese M: Modified frailty index associated with Clavien-Dindo IV complications in robot-assisted radical prostatectomies: A retrospective study. Urol Oncol 35: 425-431,2017.

21. Tsiouris A, Hammoud ZT, Velanovich V, Hodari A, Borgi J and Rubinfeld I: A modified frailty index to assess morbidity and mortality after lobectomy. J Surg Res 183: 40-46, 2013.

22. Uppal S, Igwe E, Rice LW, Spencer RJ and Rose SL: Frailty index predicts severe complications in gynecologic oncology patients. Gynecol Oncol 137: 98-101, 2015.
23. Abt NB, Richmon JD, Koch WM, Eisele DW and Agrawal N: Assessment of the predictive value of the modified frailty index for clavien-dindo grade IV critical care complications in major head and neck cancer operations. JAMA Otolaryngol Head Neck Surg 142: 658-664, 2016.

24. Augustin T, Burstein MD, Schneider EB, Morris-Stiff G, Wey J, Chalikonda S and Walsh RM: Frailty predicts risk of life-threatening complications and mortality after pancreatic resections. Surgery 160: 987-996, 2016.

25. Rolfson DB, Majumdar SR, Tsuyuki RT, Tahir A and Rockwood K: Validity and reliability of the Edmonton Frail Scale. Age Ageing 35: 526-529, 2006.

26. Folstein MF, Folstein SE and McHugh PR: 'Mini-mental state'. A practical method for grading the cognitive state of patients for the clinician. J Psychiatr Res 12: 189-198, 1975.

27. Vellas B, Guigoz Y, Garry PJ, Nourhashemi F, Bennahum D, Lauque S and Albarede JL: The Mini Nutritional Assessment (MNA) and its use in grading the nutritional state of elderly patients. Nutrition 15: 116-122, 1999.

c) (i) $\ominus$ This work is licensed under a Creative Commons Attribution-NonCommercial-NoDerivatives 4.0 International (CC BY-NC-ND 4.0) License. 